\title{
Parâmetros e tendências genéticas para pesos de várias idades em bovinos Nelore
}

\author{
Genetic parameters and trends for weight and age in Nellore
}

\author{
SILVA, Rosana Moreira da ${ }^{1 *}$; SOUZA, Júlio César de ${ }^{1,2}$; SILVA, Luiz Otávio Campos \\ $\mathrm{da}^{3}$; SILVEIRA, Maurício Vargas da ${ }^{1}$; FREITAS, José Antônio de ${ }^{4}$; MARÇAL, Mariela \\ Ferreira $^{1}$
}

\author{
${ }^{1}$ Universidade Federal de Mato Grosso do Sul, Faculdade de Medicina Veterinária e Zootecnia, Programa \\ de Pós Graduação em Ciência Animal, Campo Grande, Mato Grosso do Sul, Brasil. \\ ${ }^{2}$ Universidade Federal de Mato Grosso do Sul, Departamento de Biociência, Aquidauana, Mato Grosso \\ do Sul, Brasil \\ ${ }^{3}$ Empresa Brasileira de Pesquisa Agropecuária, Centro Nacional de Pesquisa Gado de Corte, Campo \\ Grande, Mato Grosso do Sul, Brasil. \\ ${ }^{4}$ Universidade Federal do Paraná, Departamento de Medicina Veterinária, Palotina, Paraná, Brasil. \\ *Endereço para correspondência: rosana.msilva@uol.com.br.
}

\section{RESUMO}

Objetivou-se estimar parâmetros, correlações e tendências genéticas para os pesos de animais da raça Nelore criados na região da Mata e no Agreste Nordestino aos 205 (P205), 365 (P365) e 550 (P550) dias de idade. Para realização das análises genéticas, utilizou-se da metodologia de modelos mistos. O modelo estatístico continha os efeitos aleatórios aditivos diretos, aditivos maternos, ambiente permanente e o erro; efeitos fixos de grupo contemporâneo fazenda, sexo, estação (água e seca), mês e ano de nascimento do bezerro e a covariável idade da vaca ao parto. As tendências genéticas foram estimadas pela regressão dos valores genéticos sobre o ano de nascimento dos animais. As estimativas da herdabilidade direta foram $0,13 \pm$ 0,03 para $\mathrm{P} 205 ; 0,03 \pm 0,02$ para $\mathrm{P} 365$ e $0,12 \pm$ 0,04 para P550. As correlações variaram de 0,55 a 0,85 . Os parâmetros genéticos permitem ganhos por meio da seleção e, de acordo com as correlações, a seleção para peso a determinada idade possibilitará ganhos as idades posteriores, podendo assim realizar-se seleções em idades mais jovens, apesar dos ganhos serem lentos devido aos baixos coeficientes de herdabilidade.

\section{SUMMARY}

The study aimed to was estimate the genetics parameters, correlations and trends for the weight of Nellore breed bovines at 205, 365 and 550 days of age. Genetics analysis was carried out by the methodology of mixing models, the statistical model containing the aleatory direct and maternal genetic effects, the uncorrelated maternal permanent environmental effect and the error, as fixed effects of contemporary group (farm, sex, season, water and drought, the year of birth of the calf), and the covariable age of dam. The genetic trends were estimated by linear regression of the breeding values on years of birth of the animals. Estimates of direct heritabilities were $0.13 \pm 0.03$ to W205; $0.03 \pm$ 0.02 to W365; and $0.12 \pm 0.04$ to W550. Genetic correlations were of 0.55 a 0.85 . The genetic parameters allow gains through selection and, according to the correlations, selection for weight gain to a certain age will allow gains weight at later ages, despite the gains are slow due to low heritability coefficients.

Keywords: genetic gains, heritabilities, zebu.

Palavras-chave: ganhos genéticos, herdabilidade, zebu. 


\section{INTRODUÇÃO}

A pecuária de corte é praticada em todo território nacional. As condições edafoclimáticas do País permitem a adaptação da atividade às peculiaridades regionais por meio de diversos sistemas de produção estruturados de forma a garantir a produtividade do rebanho, cada vez mais apoiados em programas de melhoramento genético.

A meta de programas de melhoramento genético é mudar a herança dos animais pela incorporação de novos genes capazes de aumentar a produtividade dos rebanhos. Para tanto se faz necessário o conhecimento de parâmetros genéticos (herdabilidades e correlações genéticas) que orientam a escolha dos métodos de seleção (KOOTS et al. 1994ab).

Outro ponto de suma importância dentro do programa de melhoramento genético é o acompanhamento o progresso das características ao longo do tempo, que assim viabiliza fazer interferências quando necessárias, almejando alcançar os objetivos de seleção (SANTOS, 2012). Tradicionalmente, medidas de pesos em determinadas idades servem como critérios de seleção nos programas de melhoramento de gado de corte em virtude de apresentarem herdabilidades de magnitude média a alta; também por estar diretamente ligadas ao retorno em ganho de peso, tempo de permanência do animal no rebanho; apresentar alta correlação com o produto final (a quantidade de carne) e por serem de fácil medição (LÔBO \& MARTINS FILHO, 2002; SALA et al., 2009).

Nesse sentido, objetivou-se estimar parâmetros, correlações e tendências genéticas para as características de pesos aos 205 (P205), 365 (P365) e 550 (550) dias de idade em bovinos Nelore criados na região da Mata e no Agreste Nordestino.

\section{MATERIAL E MÉTODOS}

Utilizou-se uma base de dados com 36.482 animais na matriz de parentesco, contendo 14.284; 5.941 e 3.895 informações de pesos padronizados aos 205; 365 e 550 dias de idade, respectivamente, de bovinos da raça Nelore criados na região da Mata e no Agreste Nordestino entre os anos de 1975 e 2003. Estas informações foram provenientes do Arquivo Zootécnico Nacional - Raças Zebuínas (Convênio ABCZ/EMBRAPA)

As análises preliminares para consistência dos dados e formação dos grupos de contemporâneos foram realizadas por meio do software Statistical Analysis System (SAS Institute, 2010). O modelo estatístico para característica única incluiu os efeitos fixos de grupo de contemporâneos, sendo estes compostos por sexo, fazenda, estação (outubro a março $=$ chuvosa, e março a setembro $=$ seca) e ano de nascimento dos animais (1975 a 2003); idade da mãe ao parto como covariável (linear e quadrática), e os efeitos aleatórios de animal (genético direto), mãe (genético materno), de ambiente permanente e ambiental (residual). Os grupos contemporâneos formados tinham, no mínimo, nove indivíduos, além de touros com mínimo cinco filhos. Na Tabela 1, é apresentada uma estatística descritiva dos dados avaliados.

Para obter as estimativas dos parâmetros genéticos, com base em análises uni e bicaráterísticas, empregou-se a modelo animal, utilizando o aplicativo Multiple Traits Derivate Free Restrict Maximum Likelihood, MTDFREML, de Boldman et al. (1995). As interações foram interrompidas quando a variância do "simplex" foi menor que $10^{-9}$. 
Tabela 1. Estatística descritiva para peso ao desmame (P205), peso ao ano (P365) e peso ao sobreano (P550) de um rebanho Nelore

\begin{tabular}{lcccccc}
\hline Característica & $\mathrm{N}$ & Touros & Vacas & G C & Média \pm DP $(\mathrm{kg})$ & $\mathrm{CV}(\%)$ \\
\hline P205 & 14.284 & 1.534 & 7.071 & 656 & $176,65 \pm 48,97$ & 27,72 \\
P365 & 5.941 & 887 & 3.845 & 620 & $273,68 \pm 63,07$ & 23,04 \\
P550 & 3.895 & 688 & 2.782 & 553 & $366,94 \pm 111,56$ & 30,40 \\
\hline
\end{tabular}

$\mathrm{N}$ = número de observações; $\mathrm{GC}$ = grupos de contemporâneos; $\mathrm{CV}=$ coeficiente de variação.

A cada convergência o programa era reiniciado utilizando valores iniciais obtidos na análise anterior até que o valor $-2 \log \mathrm{L}$ não reduzisse mais, alcançando a convergência global. $\mathrm{Na}$ forma matricial, o modelo utilizado foi:

$y=X \beta+Z g+M m+W p e+e$, onde:

$\mathrm{y}=$ Vetor das variáveis dependentes; $\beta$ $=$ Vetor dos efeitos fixos, incluindo grupo de contemporâneos (ano de nascimento, estação de nascimento, sexo e fazenda); $X=$ Matriz de incidência associando $\beta$ com $\mathrm{y} ; \mathrm{g}=$ Vetor dos efeitos aleatórios de valor genético aditivo direto do animal; $\mathrm{Z}=$ Matriz de incidência associando g com $\mathrm{y} ; \mathrm{m}=$ Vetor dos efeitos aleatórios de valor genético aditivo materno; $\mathrm{M}=$ Matriz de incidência associando $\mathrm{m}$ a $\mathrm{y}$; pe $=$ Vetor dos efeitos aleatórios de ambiente permanente da vaca; $\mathrm{W}=$ Matriz de incidência associando $p$ com $\mathrm{y} ; \mathrm{e}=$ Vetor dos efeitos residuais.

As estimativas das tendências genéticas diretas e maternas para a característica de $\mathrm{P}(205), \mathrm{P}(365)$ e $\mathrm{P}(550)$ foram obtidas por meio da análise de regressão do valor genético médio do animal sobre o ano de nascimento, utilizando o procedimento PROC REG, do programa SAS (SAS Institute, 2010).

\section{RESULTADOS E DISCUSSÃO}

As estimativas dos componentes de variância e das herdabilidades estão apresentadas na Tabela 2. As estimativas de herdabilidades foram de baixa magnitude, indicando pouca participação da variação genética aditiva na transmissão das características, de forma que a expressão destas está fortemente ligada a efeitos não genéticos.

O peso, aos 205 dias de idade, apresenta grande importância ao produtor por determinar até $50 \%$ do peso do animal aos seus 550 dias de idade (MALHADO et al., 2005). De acordo com a média apresentada neste trabalho, o P205 assume $48,14 \%$ da média do peso aos 550 dias de idade, mostrando a importância desta característica para o desempenho do animal em idades futuras.

Contudo, os estudos genéticos para P205 demonstraram reduzido coeficiente de herdabilidade direta $(0,13)$, valor inferior aos observados por Gonçalves et al. (2011), Laureano et al. (2011) e Silva et al. (2012), que obtiveram valores iguais a 0,$60 ; 0,23$ e 0,24 , respectivamente. Porém, Souza et al. (2011) encontraram $\mathrm{h}^{2}$ de 0,14 , valor próximo ao estimado neste trabalho, sugerindo que o valor fenotípico não deva ser diretamente utilizado como critério de seleção, visto que muito da variação do peso a esta 
idade, entre os animais, não é decorrente do efeito genético direto.

As herdabilidades para P365 $(0,02)$ e P550 (0,12), também com reduzidos valores, seguem o mesmo comportamento discutido para P205, estando menores que valores descritos por Garneiro et al.
(2010), sendo estes 0,31 e 0,26 para P365 e P550, respectivamente, em estudos com a raça Nelore. Gonçalves et al. (2011) e Silva et al. (2012) também estimaram, para animais da raça Nelore, herdabilidades superiores as determinados neste estudo para P365 e P550.

Tabela 2. Componentes de (co)variância, herdabilidades e componentes ambientais para peso aos 205, 365 e 550 dias de idade de animais da raça Nelore criados na região da Mata e Agreste Nordestino

\begin{tabular}{lccc}
\hline Item & P205 & P365 & P550 \\
\hline$\sigma^{2}{ }_{a}$ & 226,10 & 79,84 & 922,18 \\
\hline$\sigma^{2}{ }_{\mathrm{m}}$ & 59,03 & 164,78 & 144,71 \\
$\sigma_{\mathrm{am}}$ & $-77,36$ & 0,00 & 0,00 \\
$\mathrm{~h}^{2}{ }_{\mathrm{a}}$ & $0,13 \pm 0,03$ & $0,03 \pm 0,02$ & $0,12 \pm 0,04$ \\
$\mathrm{~h}^{2}{ }_{\mathrm{m}}$ & $0,03 \pm 0,02$ & $0,06 \pm 0,04$ & $0,02 \pm 0,06$ \\
$\mathrm{c}$ & $0,41 \pm 0,01$ & $0,54 \pm 0,05$ & $0,71 \pm 0,06$ \\
$\mathrm{e}^{2}$ & $0,84 \pm 0,02$ & $0,91 \pm 0,02$ & $0,86 \pm 0,04$ \\
\hline$\sigma^{2}{ }_{\mathrm{a}}=$ variância genética aditiva; & $\sigma_{\mathrm{m}}^{2}=\mathrm{variância}^{2}$ genética materna; $\sigma_{\mathrm{am}}=$ covariância aditiva materna; \\
$\mathrm{h}^{2}{ }_{\mathrm{a}}=$ herdabilidade do efeito aditivo direto; $\mathrm{h}_{\mathrm{m}}^{2}=$ herdabilidade do efeito materno; $\mathrm{c}=$ ambiente permanente; \\
$\mathrm{e}^{2}=$ proporção da variância ambiental sobre a variância fenotípica.
\end{tabular}

Observa-se a maior proximidade ao valor nulo $(0,02)$ da herdabilidade estimada para característica P365, de modo semelhante ao encontrado por Souza et al. (2011) para P550 (0,09), indicando que, no caso de uma mesma intensidade de seleção, a resposta para $\mathrm{P} 365$ deverá ser inferior as respostas geradas pelas demais características avaliadas.

De modo geral, segundo Silva et al. (2012), as estimativas, embora não possam ser consideradas altas, indicam que é possível ser alcançado ganho genético por seleção para todas as características avaliadas.

As estimativas de herdabilidade aditiva materna para P205 $(0,03)$; P365 $(0,06)$; P550 $(0,02)$ demonstram que o efeito materno teve pouca importância, principalmente, no peso ao sobreano que, segundo Ribeiro et al. (2001), demonstra a pequena dependência dos bezerros em relação às suas mães, sugerindo que a variação fenotípica total para essas características depende, em grande proporção, do efeito genético aditivo direto. No entanto, apesar da menor influência dos efeitos maternos em pesos após desmame, estes continuam presentes (LAUREANO et al., 2011).

Segundo Castro-Pereira et al. (2007), a dificuldade em melhorar a habilidade materna para essas características pela seleção é devido as magnitudes menores que as encontradas para as herdabilidades direta, além do antagonismo genético entre os efeitos diretos e efeitos maternos em razão da alta variância aditiva e da baixa variância materna. $\mathrm{O}$ manejo adotado para reposição de fêmeas pode contribuir para esse comportamento, principalmente quanto a repetibilidade das matrizes dentro do rebanho evidenciada pelos resultados de ambiente permanente maternal. 
As estimativas das correlações genéticas foram positivas e com média magnitude $(0,55)$ entre as características P205 e $\mathrm{P} 550$, à alta magnitude com valores de 0,80 (P365 e P550), e 0,85 (P205 e P550). As maiores correlações estimadas nesse estudo estão associadas com o P205, indicando que os genes responsáveis por maiores pesos aos 205 dias de idade são os mesmos atuantes nas demais idades. Dessa forma pode-se dizer que a seleção para o aumento do peso em idade mais jovem leva ao aumento do peso adulto, o que está de acordo com Mercadante et al. (2004), onde afirmam que, em gado de corte, as estimativas de correlações genéticas entre pesos em diferentes idades são positivas e de magnitude moderada a alta.

Boligon et al. (2009), estudando bovinos de corte, estimaram uma correlação de
$82 \%$ do peso à desmama e do peso ao sobreano. Resultados com semelhante comportamento foram estimados por Baldi et al. (2010), com correlações de 0,36 (PN - P30 meses) a 0,98 (P18 meses - P24 meses).

A mudança genética média anual foi de 0,207, 0,069 e -0,142kg por ano e para P205, P365 e P550, respectivamente (Figura 1), sendo significativas $(P<0,001)$ para todas as características.

Esse desempenho representa incrementos de 0,12 e $0,03 \%$ nas médias anuais de P205 e P365, respectivamente, e uma diferença de aproximadamente 5,8 e $1,93 \mathrm{~kg}$ no período de seleção estudado (28 anos) para estas características, respectivamente, enquanto que para P550 observou-se uma redução de $0,04 \%$ na média anual, dada em uma diferença negativa de $3,98 \mathrm{~kg}$ no período estudado.
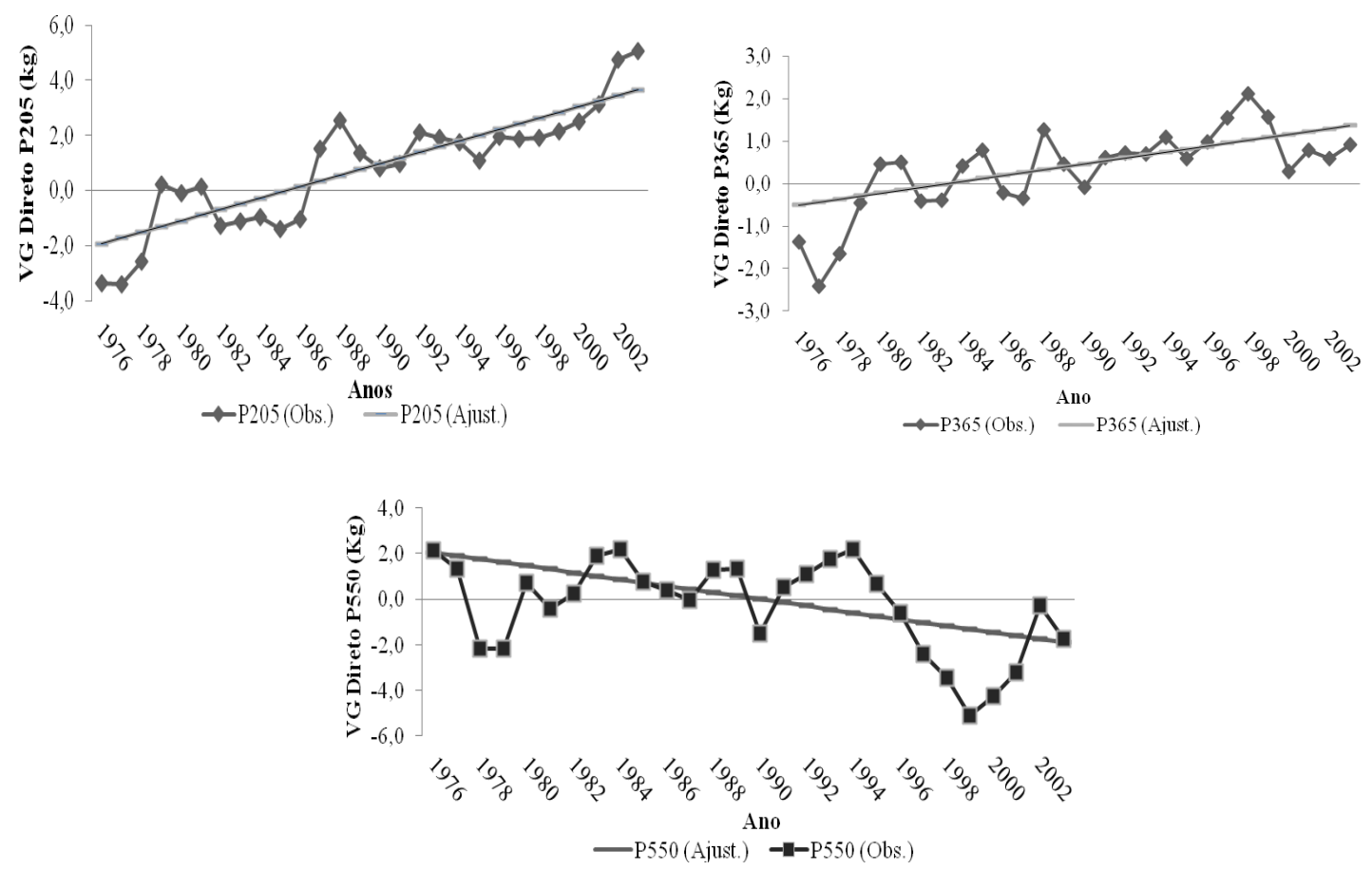

Figura 1. Tendência genética dos valores genéticos (VG) direto, observados e ajustados pela equação VGD $=0.207 *$ ano -411.0465 para pesos aos $205(\mathrm{VGD}=$ $0.207 *$ ano - 411.0465; $\mathrm{R}^{2}$ : 0,92), aos 365 (VGD = 0.06900*ano - 136.84309; $\left.\mathrm{R}^{2}: 0,97\right)$ e aos 550 dias de idade $\left(\mathrm{VGD}=-0.14262 *\right.$ ano $+291.05897 ; \mathrm{R}^{2}$ : $0,92)$ 
Souza et al. (2008), avaliando bovinos Nelore criados no Pantanal e em Goiás, determinaram para P205 ganho genético direto de $0,322 \mathrm{~kg} /$ ano para rebanhos da região do Pantanal e $0,455 \mathrm{~kg} /$ ano para o rebanho de Goiás. Já Gonçalves et al. (2011) apresentaram crescente tendência para os efeitos genéticos aditivos diretos para as características P205 (0,9378kg/ano), P365 (1,7393kg/ano) e P550 (2,2212kg/ano). Em animais da raça Brangus, Silva et al. (2011) encontraram mudança genética média anual de 0,$107 ; 0,177$ e $0,217 \mathrm{~kg}$ por ano para P205; P365 e P550, respectivamente. Para a característica P550 houve uma redução de $-0,142 \mathrm{~kg}$ por ano, com picos e quedas durante os anos, o que indica ineficiência do programa de seleção durante o período, sugerindo que para essa característica não tem ocorrido pressão de seleção, consequentemente, tampouco progresso genético.

As tendências genéticas maternas apresentaram-se significativas $(\mathrm{P}<0,001)$ para todas as características. A característica P205 (Figura 2) apresentou declínio médio ao longo do período de - 0,045 $\mathrm{kg}$ por ano assim como para a característica P365, com redução anual de $-0,007 \mathrm{~kg}$ (Figura 5). Esse comportamento se deve, segundo Correa et al. (2006), à correlação negativa entre o efeito direto e efeito materno, visto que, o efeito materno diminui a medida que o efeito direto aumenta. Gonçalves et al. (2011) ressaltam que este fato é esperado, uma vez que se é dada ênfase (seleção) para as características de produção (desempenho ponderal etc.).
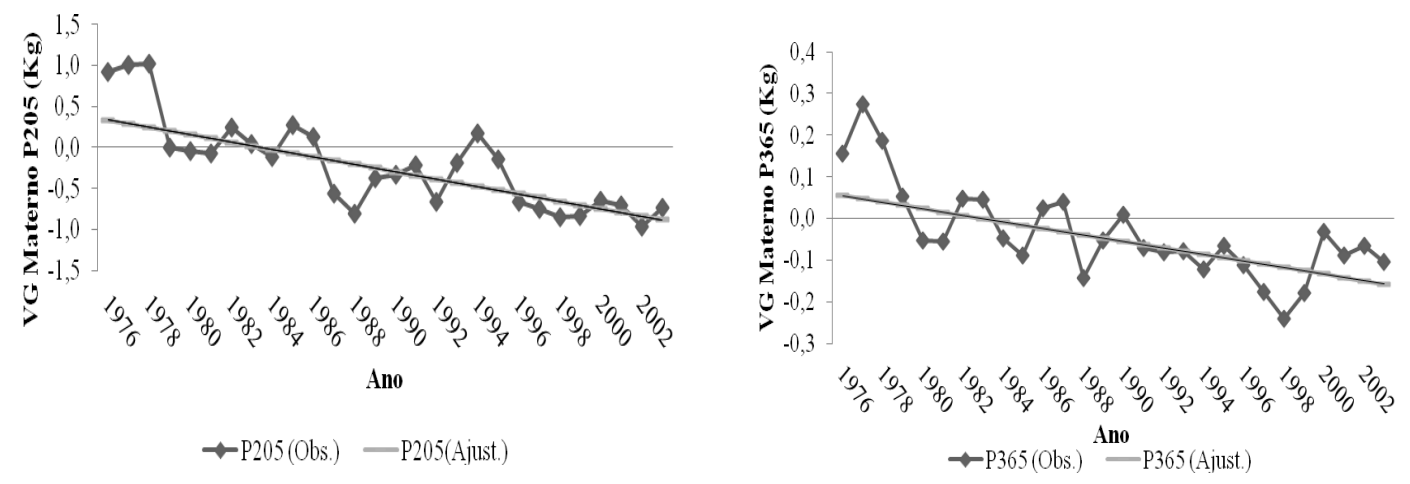

Figura 2. Tendência genética dos valores genéticos (VG) materno, observados e ajustados para pesos aos $205\left(\mathrm{VGM}=-0.045^{*}\right.$ ano $\left.+89.31329 ; \mathrm{R}^{2}: 0,96\right)$ e 365 dias de idade $\left(\mathrm{VGM}=-0.007 *\right.$ ano $\left.+15.5662 ; \mathrm{R}^{2}: 0,92\right)$

Esses resultados podem indicar inexistência de seleção para 0 componente materno para P205 e P365, o que, segundo Souza et al. (2008), pode vir a comprometer a expressão da habilidade materna, prejudicando o desempenho de futuras progênies. Resposta diferente pode-se observar para P550, em que a mudança genética materna anual foi de $0,83 \mathrm{~kg}$, correspondendo a $0,023 \%$ em relação à média da característica.

Os valores das estimativas de herdabilidade diretas indicam possibilidade de seleção, porém com progresso lento. As correlações genéticas analisadas sugerem que uma pré-seleção aos 205 dias de idade resultará em 
potencial genético para maiores pesos nas idades posteriores, principalmente ao P550. As tendências genéticas revelaram que a seleção praticada no rebanho não foi enfocada no aumento no peso dos animais, apresentando-se baixas e, no caso de P550, decrescente, não obtendo ganhos genéticos expressivos ao longo dos 28 anos estudados.

\section{REFERÊNCIAS}

BALDI, F.; ALENCAR, M.M; ALBUQUERQUE, L.G. Estimativas de parâmetros genéticos para características de crescimento em bovinos da raça Canchim utilizando modelos de dimensão finita. Revista Brasileira de Zootecnia, v.39, n.11, p.2409-2417, 2010.

BOLDMAN, K.G.; KRIESE, L.A.; VAN VLECK, L.D.; VAN TASSELL, C.P.; KACHMAN, S.D. 1995. A Manual for use of MTDFREML, a set of programs to obtain estimates of variances and covariances [DRAFT]. Lincoln: Department of Agriculture, Agricultural Research Service. 120p.

BOLIGON, A.A.; ALBUQUERQUE, L.G.; MERCADANTE, M.E.Z; LÔBO, R.B. Herdabilidades e correlações entre pesos do nascimento à idade adulta em rebanhos da raça Nelore. Revista

Brasileira de Zootecnia, v.38, n.12, p.2320-2326, 2009.

CASTRO-PEREIRA, V.M.; ALENCAR, M.M.; BARBOSA, R.T. Estimativas de parâmetros genéticos e de ganhos direto e indireto à seleção para características reprodutivas e de crescimento em um rebanho da raça Canchim. Revista Brasileira de
Zootecnia, v.36, n.4, p.1029-1036, 2007. Supl. 1.

CORREA, M.B.B.; DIONELLO, N.J.L.; CARDOSO, F.F. Estimativa de parâmetros genéticos, componentes de (co)variância e tendências genéticas e fenotípicas para características produtivas pré-desmama em bovinos Devon no Rio Grande do Sul. Revista Brasileira de Zootecnia, v.35, n.3, p.997-1004, 2006. Supl.

GARNERO, A.V.; MUÑOZ, M.C.C.D.; MARCONDES,C.R.; LÔBO, R.B.; LIRA, T.; GUNSKI, R.J. . Estimação de parâmetros genéticos entre pesos pré e pós-desmama na raça Nelore. Archivos de Zootecnia, v.59, n.226, p.307-310, 2010 .

GONÇALVES, F.M.; PIRES, A.V.; PEREIRA, I.G.; GARCIA, D.A.; FARAH, M.M.; MEIRA, C.T.; CRUZ, V.A.R.Avaliação genetica para peso corporal em um rebanho Nelore.

Arquivo Brasileiro de Medicina Veterinária e Zootecnia, v.63, n.1, p.158-164, 2011.

KOOTS, K.R.; GIBSON, J.P.; WILTON, J.W. Analyses of published genetic parameters estimates for beef production traits. 1- Heritability.

Animal Breed Abstract, v.62, n.5, p.309-308, 1994a.

KOOTS, K.R.; GIBSON, J.P.; WILTON, J.W. Analyses of published genetic parameters estimates for beef production traits. 2- Phenotypic and genetic correlations. Animal Breed Abstract, v.62, n.11, p.825-853, 1994b.

LAUREANO, M.M.M.; BOLIGON, A.A.; COSTA, R.B.; FORNI, S.; SEVERO, J.L.P.; ALBUQUERQUE, L.G. Estimativas de herdabilidade e tendências genéticas para características 
de crescimento e reprodutivas em bovinos da raça Nelore. Arquivo Brasileiro de Medicina Veterinária e Zootecnia, v.63, n.1, p.143-152, 2011.

LÔBO, R.N.B.; MARTINS FILHO, R. Avaliação de métodos de padronização dos pesos corporais as idades de 205 , 365 e 550 dias. Revista Brasileira de Zootecnia, v.31, n.4, p.1695-1706, 2002.

MALHADO, C.H.M.; CARNEIRO, P.L.S.; MARTINS FILHO, R.

Tendência e parâmetros genéticos para o peso aos 205 dias de idade em bovinos da raça Nelore Mocho no estado da Bahia. Revista Cientifica de Produção Animal, v.7, n.2, p.28-34, 2005.

MERCADANTE, M.E.Z.; RAZOOK, A.G.; CYRILLO, J.N.S.G.; FIGUEIREDO, L.A. Programa de seleção da estação experimental de zootecnia de Sertãozinho: resultados de pesquisas, sumário de touros Nelore. Nova Odessa, SP: Instituto de Zootecnia, 2004. p.35. (Boletim Cientifico, 12).

RIBEIRO, M.N.; PIMENTA FILHO, E.C.; MARTINS, G.A.; SARMENTO, J.L.R.; MARTINS FILHO,

R.Herdabilidade para efeito direto e materno de características de crescimento de bovinos Nelore no estado da Paraíba. Revista Brasileira de Zootecnia, v.30, p.1224-1227, 2001.
SALA, V.E.; ALBUQUERQUE, L.G.; MERCADANTE, M.E.Z.; BOLIGON, A.A.; BONILHA, S.F.M.Eficiência produtiva em vacas da raça Nelore. Boletim da Indústria Animal, v.66, n.2., p.107-113, 2009.

SANTOS, G.C.J.; LOPES, F.B.; MARQUES, E.G.; SILVA, M.C.; CAVALCANTE, T.V.; FERREIRA, J.L. Tendência genética para pesos padronizados aos 205, 365 e 550 dias de idade de bovinos Nelore na região norte do Brasil. Acta Scientiarum Animal Sciences, v.34, n.1, p.97-101, 2012.

SAS Institute. Statistical analysis system: user's guide: statistics. Cary, NC, 2010.

SILVA, J.A.V.; MARCELO, E.T.; RIBEIRO, C.B.; MAIORANO, A.M.; CURI, R.A.; OLIVEIRA, H.N.; MOTA, M.D.S. Análise genética de características de crescimento e perímetro escrotal em bovinos da raça Brangus. Pesquisa Agropecuária Brasileira, v.47, n.8, p.1166-1173, 2012.

SOUZA, J.C., DOSKA, M.C., SILVA, L.O.C.; GONDO, A.; RAMOS, A.A.; MALHADO, C.H.M.; SANTOS, I.W.; FREITAS, J.A.; FERRAZ FILHO, P.B.; SERENO, J.R.B.Interacción genótipo $\mathrm{x}$ ambiente sobre El peso al destete de bovinos Nelore en Brasil. Archivo de Zootecnia, v.57, n.218, p.171-177, 2008.

Data de recebimento: $28 / 06 / 2012$

Data de aprovação: 29/11/2012 